\title{
The use of transcatheter aortic valve replacement vs surgical aortic valve replacement for the treatment of aortic stenosis
}

This article was published in the following Dove Press journal:

Research Reports in Clinical Cardiology

25 August 2015

Number of times this article has been viewed

\author{
Hanna A Jensen \\ Lillian L Tsai \\ Vinod H Thourani \\ Division of Cardiothoracic Surgery, \\ Joseph B Whitehead Department of \\ Surgery, Structural Heart and Valve \\ Center, Emory University School of \\ Medicine, Atlanta, GA, USA
}

\begin{abstract}
Severe aortic stenosis (AS) is associated with considerable morbidity and mortality and is increasing in prevalence as the global population increases. Since AS primarily affects the elderly, many of these patients have comorbidities that make them poor candidates for the gold standard treatment for AS, surgical aortic valve replacement. Transcatheter aortic valve replacement has emerged as a novel technology for the management of AS in higher risk patients over the past decade. Randomized trials have established the safety and efficacy of transcatheter aortic valve replacement, and the medical community has rallied to identify the patients who are most suitable for this transformative treatment. This review focuses on outlining the key procedural differences, describing the unique challenges of both operations, and finally assessing and comparing outcomes both on a general level and in challenging patient subgroups.

Keywords: aortic valve replacement, transcatheter aortic valve replacement, surgical aortic valve replacement
\end{abstract}

\section{Introduction}

Aortic stenosis (AS) is the most common acquired valve disease in elderly patients with a prevalence of $\sim 2.8 \%$ in those aged 75 years or older, ${ }^{1}$ a population that is globally increasing at a rapid rate. Treated with medical therapy alone, severe AS is associated with a $50 \%-70 \%$ mortality rate within 2 years. ${ }^{2}$ Currently when replacement of the aortic valve is indicated, two mainstream treatment pathways are available: 1) the traditional, "gold standard" surgical aortic valve replacement (SAVR) with a biological or mechanical prosthesis and 2) transcatheter aortic valve replacement (TAVR). Several intriguing variations of SAVR with minimally invasive/sutureless techniques exist and are practiced worldwide, but those are outside of the scope of this review, which will focus on outlining the procedures and data acquired to date about the outcomes and patient selection for SAVR and TAVR, each with their unique set of advantages and challenges.

\section{Procedural overview}

Briefly, SAVR is performed through a midline sternotomy with cardiopulmonary bypass and cross-clamping of the aorta. A mechanical or a tissue valve is sutured in place after excision of the native valve and decalcification of the aortic annulus. The patient is always under general anesthesia, and the procedure is performed in an operating room fit for open-heart surgery. ${ }^{3}$ While operative techniques have remained fairly consistent throughout the last decade, the most notable operative trend is the increasing use of bio-prosthetic valves, which are now implanted in approximately
Correspondence: Vinod H Thouran Division of Cardiothoracic Surgery, Emory Hospital Midtown, Structura Heart and Valve Center, Medical Office Tower 6th Floor, 550 Peachtree Street NE, Atlanta, GA 30308, USA

Tel + I 4046862513

$\mathrm{Fax}+\mathrm{I} 4046864959$

Email vthoura@emory.edu 
$80 \%$ of patients requiring SAVR. ${ }^{4}$ This likely reflects the increasing age in the operated population as surgeons wish to avoid anticoagulation, and the slightly improved durability of mechanical valves does not play a critical role in clinical decision-making for the elderly cohort. ${ }^{5}$

TAVR can be performed via several access routes including the following:

- Femoral artery (percutaneous transfemoral or TF-TAVR)

- Apex of the left ventricle (transapical or TA-TAVR performed through a left mini-thoracotomy)

- Distal ascending aorta (transaortic or TAo-TAVR performed through a upper mini-sternotomy)

- Axillary artery (trans-subclavian or TS-TAVR through a surgical cut-down)

- Right common carotid artery (transcarotid or TC-TAVR through an incision in the lower neck)

- Femoral vein (crossed over to arterial side through an aortocaval fistula referred to as transcaval or TCvl-TAVR).

In some cases, TF-TAVR can be performed in the catheterization laboratory with conscious sedation, ${ }^{6}$ and patients can be eligible for a fast-track hospital stay. ${ }^{7}$ However, the utilization of non-transfemoral (also known as alternative access) TAVR approaches currently necessitates general anesthesia and at least a hybrid operating room setup.

While several novel valves are currently undergoing development and clinical testing, two TAVR valves are commercially available in the USA: the balloon-expandable SAPIEN system (Edwards Lifesciences, Irvine, CA, USA) and the self-expanding CoreValve prosthesis (Medtronic, Inc., Minneapolis, MN, USA). The transapical, antegrade approach is currently available only with the SAPIEN valve, while the transaortic techniques can be used by both valve systems. Generally, the trans-subclavian technique is utilized with the self-expanding prosthesis. The current generation of the balloon-expandable valves is available in sizes $23 \mathrm{~mm}$, $26 \mathrm{~mm}$, and $29 \mathrm{~mm}$, and the self-expanding valve comes in sizes $23 \mathrm{~mm}, 26 \mathrm{~mm}, 29 \mathrm{~mm}$, and $31 \mathrm{~mm}$.

Both transcatheter heart valve systems have been available in Europe for over a decade. In the USA, the EdwardsSAPIEN valve gained Food and Drug Administration (FDA) approval in 2011 and the Medtronic CoreValve in 2014. The operative details of TAVR procedure have been published previously. ${ }^{8}$

\section{Patient selection - what is high risk?}

Appropriate patient selection is the prerequisite of success for any surgical procedure. Currently, TAVR is indicated for patients with severe symptomatic AS who are considered either ineligible or high risk for SAVR due to comorbidities or technical issues. An evaluation of the Society of Thoracic Surgeons Adult Cardiac Surgery Database notes that $\sim 6 \%-7 \%$ of patients presenting for SAVR in North America are considered high-risk patients. ${ }^{4,9}$

The difficulty in drawing distinct global guidelines for high-risk patients is partially due to the variability in determining what defines "high risk". The most common tools in helping to guide decision-making are the standardized surgical scoring systems, which solicit an estimated risk of operative mortality for each individual patient. These are the Society of Thoracic Surgeons Predicted Risk of Mortality (STS PROM) and the European System for Cardiac Operative Risk Evaluation II (EuroSCORE II), which is an updated version of the traditional logistic EuroSCORE. Although primarily designed to predict the risk of 30-day mortality after SAVR based on a number of demographic and procedural variables, they are widely used to classify patients prior to transcatheter interventions, and both have shown to be adequately and significantly calibrated in association with TAVR. ${ }^{10,11}$ The US national Transcatheter Valve Therapies registry is also expected to release a short-term TAVR-specific risk model soon.

In the landmark PARTNER Trial preceding the FDA approval of the balloon-expandable valve in the USA, a patient was considered high risk if the STS PROM score was $10 \%$ or greater for death and the surgeons' assessment of the mortality risk was $>15 \%$ but $<50 \% .{ }^{12}$ Similarly, in the pioneering pivotal trial of the self-expanding valve, patients were considered to be at increased surgical risk if two cardiac surgeons and one interventional cardiologist at the investigative site estimated that the risk of death within 30 days after surgery was $15 \%$ or more, but the risk of death or irreversible complications within 30 days after surgery was $<50 \%{ }^{13}$ Beyond these large randomized prospective trials, studies have used criteria such as logistic EuroSCORE $>15 \%{ }^{14}$ or $>20 \%,{ }^{15-17}$ EuroSCORE of $9 \%$ or greater, ${ }^{18,19}$ STS PROM score $>15 \%{ }^{20}$ or $>8 \%,{ }^{9}$ or age $>75$ years $^{17,21}$ or 80 years $^{14}$ as definitions of high surgical risk.

\section{Frailty}

It should be noted that neither the STS PROM nor the EuroSCORE II comprehensively cover a variety of factors often considered very important for the clinical team such as patients' functional status and frailty. ${ }^{22}$ The biological syndrome of frailty is characterized by diminished physiological reserve and weakened ability to respond to stressors ${ }^{23}-\mathrm{a}$ 
concept particularly relevant since nearly $40 \%$ of patients receiving aortic valve replacement are 75 years or older. ${ }^{24,25}$ Factors such as disability, mobility impairment, cognitive impairment, mood disturbance, malnutrition, polypharmacy, fall risk, and social isolation are not reflected in surgical risk scores but may have a significant impact on how a patient recovers from SAVR or TAVR. ${ }^{26}$

Currently, there are over 20 different tools proposed to identify frail patients. Several studies have shown that frailty, defined by various metrics, is associated with higher postoperative early and late mortality after cardiac surgery. ${ }^{27,28}$ Stortecky et al have shown that Multidimensional Geriatric Assessment-based risk scores can predict all-cause mortality and major adverse cardiovascular and cerebral events in elderly patients undergoing TAVR, ${ }^{29}$ and others have suggested that frailty is predictive of 1-year, but not 30-day mortality in TAVR patients. ${ }^{30-32}$

Frail elderly patients may have smaller gains in functional status after TAVR or SAVR, and they may require multidisciplinary interventions, such as nutrition consultation and exercise-training programs, to achieve desired outcomes. ${ }^{33}$ Thus, it is not uncommon today for surgeons and cardiologists to consult geriatricians when frail or extremely elderly patients are considered for TAVR. ${ }^{34}$

Thus, while the standardized scores are used as a guideline, the definitive operative decision for SAVR or TAVR is made in an individual institution by a specialized multidisciplinary heart team, a process that is challenging to standardize across surgical programs.

The intricacies of patient selection to SAVR or TAVR and the key role of the heart team have been expertly explored in a recent review by Agarwal et al, and key steps are summarized in Figure $1 .{ }^{35}$ The wide variety in the criteria of high risk not only explains why an exact threshold that defines whether a patient is more suitable for TAVR than SAVR has not been defined, but also highlights the complex decision-making process that each institution must complete in this cohort of often old, frail, and multicomorbid patients.

\section{Modern SAVR}

The first SAVR was performed in the $1960 \mathrm{~s}^{36}$ and remains the gold standard treatment for AS. The quest for disruptive technology such as TAVR stemmed from the fact that an increasing population of patients with severe, symptomatic AS were considered to be ineligible for SAVR due to the relatively high-risk profile of open-heart operations. In the early 2000s, the operative mortality for SAVR was reported as greater than $8 \% .^{37}$

Today, $\sim 20,000$ patients undergo first-time isolated SAVR in the USA annually and $80 \%$ of them are classified as "low risk" for the procedure. Contemporary operative mortality for this surgical cohort has been reported as $2.4 \%-2.8 \%(1.7 \%$ for the low-risk patients, $5.4 \%$ for the medium-risk patients, and $11.9 \%$ for the high-risk patients) ${ }^{4,38}$ While the number
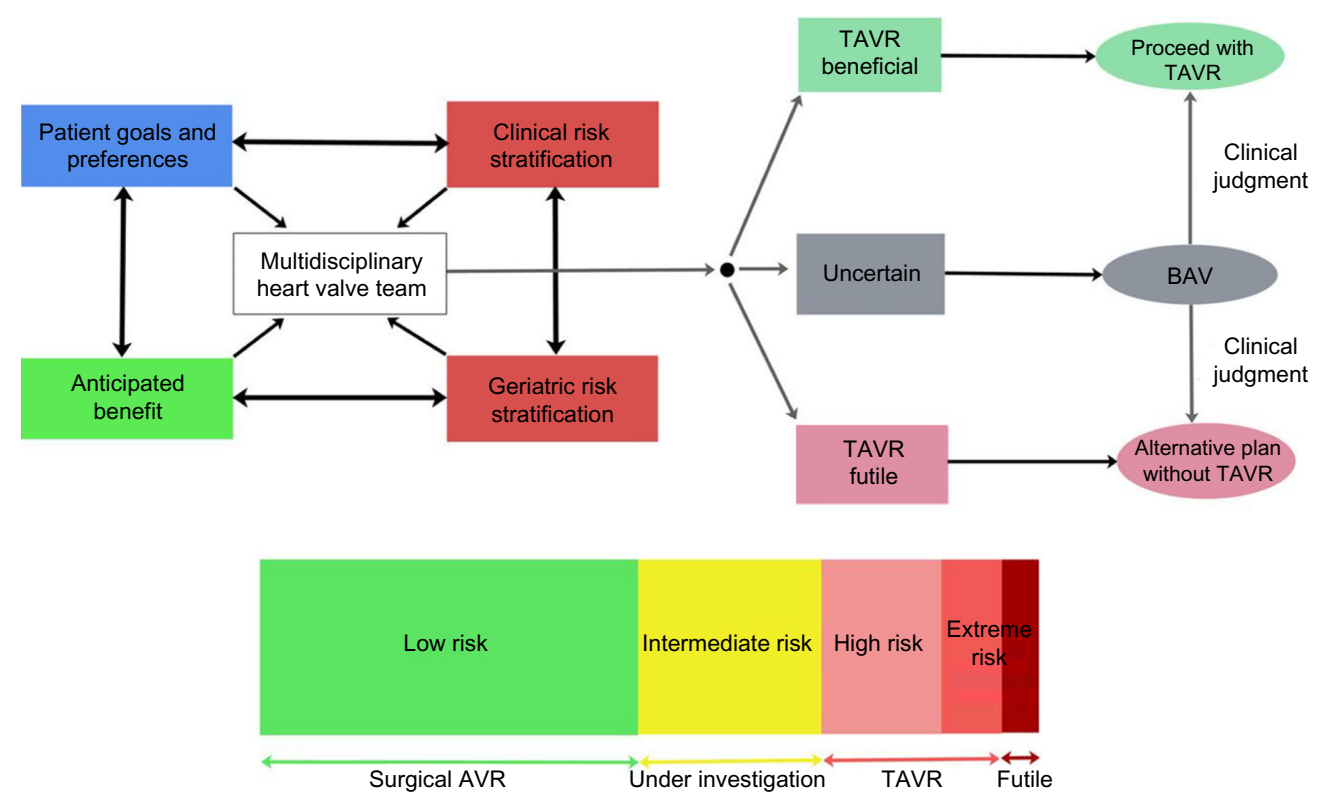

Figure I Patient Risk Algorithm.

Note: Reproduced from Agarwal S, Tuzcu EM, Krishnaswamy A, et al. Transcatheter aortic valve replacement: current perspectives and future implications. Heart. 20 I 5; I I (3): 169-177. Copyright (C) 2015, with permission from BMJ Publishing Group Ltd. ${ }^{35}$

Abbreviations: TAVR, transcatheter aortic valve replacement; BAV, balloon valvuloplasty; AVR, aortic valve replacement. 
of medium- and high-risk patients undergoing SAVR has increased in the recent decade, the overall operative mortality has not risen, most likely indicating improvements in modern pre-, per-, and postoperative care. ${ }^{4}$

Operative morbidity associated with SAVR has remained very similar throughout the last decade with atrial fibrillation quoted as the most common complication $(\sim 15 \%-25 \%)$, followed by prolonged ventilation $(10 \%)$, renal failure $(4 \%)$, reoperation for bleeding (4\%-6\%), pneumonia (3\%), stroke $(1.5 \%-1.8 \%)$, multiorgan failure $(1 \%)$, and deep sternal infection $(0.3 \%))^{4,38}$

As an open heart procedure utilizing cardiopulmonary bypass, SAVR, rather unsurprisingly, has been associated with more bleeding complications than its less invasive TAVR counterpart. ${ }^{39}$ Analysis from the PARTNER Trial cohort indicated that not only was bleeding in association with SAVR two to three times more frequent than in TAVR, but it was also more severe resulting in higher rates of kidney injury as well as 30-day and 1-year mortality. ${ }^{39}$ Perhaps due to hemodilution in the cardiopulmonary bypass circuit, patients with baseline anemia are especially likely to require transfusion, and the SAVR cohort has been shown to receive more red blood cell units than TAVR patients overall. ${ }^{14,39}$ The adverse effects of preoperative anemia, operative bleeding, and transfusion are complex and remain poorly understood in both SAVR and TAVR.

Sternotomy coupled with utilization of cardiopulmonary bypass is known to elicit a systemic inflammation reaction (SIRS) and render the patient susceptible to potential coagulation perturbations. Intuitively, SIRS occurs more commonly after SAVR than TAVR and has the greatest effect on mortality in patients with diabetes, who are already in a pro-inflammatory state prior to the procedure. Despite varying mechanisms, severe SIRS is associated with a higher mortality after SAVR, while a similar association has not been found in TAVR. ${ }^{40}$

\section{TAVR and common complications}

The overall 30-day mortality after TAVR has been reported to be $3.4 \%-12.7 \%$ in large multicenter registries and randomized controlled trials (RCTs). ${ }^{35}$ Traditionally, the most common complications are identified in the original RCTs as vascular complications $(5.9 \%-30.0 \%)$, bleeding $(9 \%-28 \%)$, paravalvular regurgitation $(6.7 \%-12 \%)$, new-onset atrial fibrillation (1.0\%-9.0\%), rhythm disturbances requiring a pacemaker $(3.4 \%-19.8 \%)$, and stroke $(2.3 \%-6.7 \%) .^{2,12,13}$ In the US national registry (the STS/American College of Cardiology TVT Registry, which has documented TAVRs in the USA after FDA approval), major complications included stroke $(2.0 \%)$, dialysis-dependent renal failure (1.9\%), and major vascular injury $(6.4 \%) .{ }^{41}$

It is important to note that the complication rates may significantly vary based on the TAVR approach used, mostly determined by the intrinsic patient factors that assign the patient into each access group. For example, a recent metaanalysis showed that post procedure acute kidney injury (AKI) and the need for renal replacement therapy are significantly lower when the patient is operated with the TF access compared with the TA approach. ${ }^{42}$ The key differences in patients and outcomes between TF and alternative access TAVR are topics that are extensively discussed elsewhere, ${ }^{41,43}$ whereas the following section focuses on typical TAVR complications in the patient cohort as a whole.

\section{Vascular complications and bleeding}

Vascular complications are often associated with TAVR access and the manipulation of the relatively large caliber delivery sheaths in more peripheral vessels, or valve oversizing and over-inflation (in the case of the balloon expandable valve) in the unforgiving aortic root. Although the very first TAVR studies reported vascular complication rates as high as $20 \%,{ }^{39}$ modern data quote incidence as low as $1 \%$ in selected populations, ${ }^{44}$ most likely reflecting the learning curve and device development of TAVR. It is anticipated that within the next few years, the majority of the transfemoral sheaths will be smaller than $16 \mathrm{~F}$.

Just like in SAVR, procedural bleeding events after TAVR are frequent and have been shown to be associated with worse prognosis. ${ }^{45,46}$ Currently, major life-threatening bleeds occur in $-3.5 \%-13.6 \%$ of the TAVR overall population. ${ }^{13,41}$ They are often related to the technical challenges of vascular complications, but the phenomena are not synonymous. A recent study also demonstrated a $6 \%$ incidence of late bleeding complications ( $\geq 30$ days after TAVR), mostly of gastrointestinal tract and neurological origin. ${ }^{47}$ These late bleeds resulted in a fourfold increase in late mortality, a significant finding especially given that most TAVR patients are on dual antiplatelet therapy for the first 6 postoperative months.

\section{Paravalvular leakage}

Paravalvular leakage (PVL) occurs when the replacement valve is not tightly sealed within the aortic annulus, often due to either suboptimal sizing or positioning or heavy calcification in the native valve and annulus. This complication is mostly unique to TAVR, where the native valve leaflets 
(instead of being excised like in SAVR) are displaced by the prosthetic valve, potentially affecting the anchoring of the valve system. Many European registries along with the PARTNER cohort have demonstrated an association with more than mild PVL and mortality after TAVR. ${ }^{12,48-50}$ Since these detrimental effects were initially noted in the medical community, several adjustments have been made in TAVR protocols worldwide to minimize PVL. It is now routine practice to use computed tomography imaging in addition to echocardiography when determining the appropriate size for the prosthetic valve. Moreover, the intraoperative use of balloon-sizing can be performed in cases where there is discrepancy between the computed tomography scans and echocardiography. ${ }^{51,52}$ Immediate perioperative balloon dilatation to minimize PVL is routinely undertaken if PVL is discovered during the operation. In those implantations that are noted to be too ventricular or aortic, a second TAVR valve prosthesis can be inserted.

The challenge of PVL can be partially overcome by technology and device development, as well as operator experience. The most recent reports of the third-generation SAPIEN 3 (Edwards Lifesciences Inc.) are already addressing the major deficiencies of earlier valves in terms of ease of use, accuracy of positioning, and paravalvular sealing, reporting mild PVL rates of $7 \%$ compared with the traditional $10 \%-12 \%$, and no moderate leaks at 30 days after TAVR. ${ }^{53,54}$

\section{Rhythm disturbances}

New-onset conduction disturbances, particularly new left bundle branch block, occur frequently after TAVR. Rhythm disturbances may stem from the fact that the transcatheter valve deployment can affect conduction pathways, the landmarks of which are not directly visualized during the percutaneous procedure. Direct mechanical injury of the left bundle branch and inflammation created by the stent containing the valve prosthesis are also potential mechanisms. ${ }^{55}$ Conduction difficulties are most often cited with the use of the self-expandable transcatheter valve, perhaps partially due to the continued expansion of the nitinol over the ensuing days. New pacemaker requirement has been described at significantly different percentages between the two types of valves, affecting $\sim 6 \%-10 \%$ of patients who undergo TAVR with the balloon-expandable Edwards SAPIEN valve and $30 \%-83 \%$ of those operated with the self-expandable CoreValve. ${ }^{41,56,57}$ While data have not conclusively shown increased pacemaker requirement rates between TAVR with the balloon-expandable valve and SAVR, TAVR with the self-expandable valve is associated with higher postoperative pacemaker requirement rates than SAVR. ${ }^{13}$ Whether pacemaker requirement affects survival and other clinical outcomes in the mid- and long-term is unclear; however, it does appear that left ventricular (LV) function deteriorates after pacemaker placement. ${ }^{58}$

\section{Stroke}

The PARTNER trial demonstrated an increased risk of operative stroke and transient ischemic attack in the TAVR patients compared with SAVR patients. ${ }^{2,12}$ In cardiac procedures, most neurologic consequences are attributed to emboli dislodged during manipulation of atherosclerotic vessels, ${ }^{59}$ and undoubtedly, the larger first-generation devices crossing the aortic arch played a significant role in this finding. However, temporary circulatory disruptions during balloon valvuloplasty (BAV) or rapid ventricular pacing can also lead to periods of suboptimal cerebral perfusion and ischemia in association with the deployment of the balloon-expandable valve.$^{60}$ Furthermore, the BAV and deployment of the transcatheter valve can expose valvular calcium deposits, tissue factor, and thrombin reserves from the diseased native valve into the circulation, triggering coagulation cascades and platelet activation leading to increased thrombogenicity, ${ }^{61}$ accounting for some of the neurological challenges in TAVR.

Modern comparisons have, however, largely mitigated the difference in stroke risk in TAVR compared with SAVR, and it is likely that the aforementioned technology development leading to smaller devices manipulated in the aortic arch has neutralized the risk profile between the interventions. In a recent study specifically examining neurological outcomes in patients with AS, there were no differences in overall neurologic injury, cerebral embolic load, ischemic lesions, and oxygen desaturation in patients undergoing SAVR or TAVR. ${ }^{62}$

For both SAVR and TAVR, it is extremely interesting to see if the embolic protection devices influence the horizon of neurological outcomes. Since the proof of concept for embolic protection devices in the setting of TAVR was demonstrated in $2010,{ }^{63}$ preclinical and early clinical data have shown considerable promise for these devices in terms of technical success and safety. Aiming to reduce cerebral burden of embolic debris by either capturing or deflecting embolized material preventing access to the supra-aortic-cerebral trunks, these devices can be utilized in both SAVR and TAVR settings. With three different embolic protection devices already in clinical phase, we should expect results from larger trials soon. ${ }^{64}$ 


\section{Comparison of TAVR and SAVR data - caution advised}

Compared with the very established SAVR, TAVR technology is following not only operative and institutional learning curves, but is also affected by the rapid development of the valves themselves, as well as the accompanying delivery systems. Thus, the largest cohorts reported to date either span a variety of technologies or exclusively focus on firstgeneration devices, and this should be noted when analyzing outcomes.

Of further consideration, the route used for TAVR access is not insignificant. TF-TAVR, as the most minimally invasive method, is widely recognized as the primary choice for any patient. If a patient is eligible for TF-TAVR, very few institutions would consider alternative access. Thus, it is generally agreed that alternative access (non-TF) patients are inherently of higher risk than their TF counterparts. The introduction and adoption of the various alternative access methods have been fluid and institution-driven, with heavy reliance on operator preference and expertise. As an example, in a very demonstrative report from a highvolume US TAVR center, the 1-year mortality of TA-TAVR patients dropped from $16.9 \%$ to $4.7 \%$ when the investigators compared the first 3 years in the TAVR experience with the latest 3 years. ${ }^{65}$

As illustrated earlier, comparing results between SAVR and TAVR is peppered with potential landmines the reader should aim to avoid. We urge that the following is kept in mind when analyzing data that compares SAVR and TAVR:

1. SAVR has been performed for $>50$ years, TAVR for approximately a decade.

2. Device design and development has been rapid in TAVR, with new generations of valves and delivery technology emerging every year.

3. TAVR operative technique has also evolved almost on an annual basis; there are now six recognized access routes, often denoting different patient populations, each with their own learning curves.

4. The boundaries between low-, intermediate-, and highrisk patients are being crossed and transformed in both SAVR and TAVR populations. Patient selection and definition of eligibility for either procedure are not universally standardized, but generally, in data published thus far, TAVR “as treated" patients are a considerably higher risk population than SAVR.

The non-inferiority and similarity in adjusted mortality outcomes have been thoroughly shown in SAVR vs TAVR. In a recent meta-analysis of 39 statistically robust studies comparing SAVR and TAVR (three RCTs, ten propensity-scorematched studies, five case-matched studies, and two adjusted studies) comprising of 13,130 patients, it was concluded that when adjusted for the baseline patient characteristics, SAVR and TAVR have a similar risk for 30-day mortality, stroke, myocardial infarction (MI), and AKI. However, SAVR patients required transfusion more often and had a higher incidence of postoperative new-onset atrial fibrillation (AF) than TAVR patients. On the other hand, patients who underwent TAVR had a higher risk of developing moderate or severe aortic regurgitation, primarily paravalvular. ${ }^{66}$ A very recent $1,000+$ patient real world dataset supported these findings as the authors demonstrated no difference in mortality between patients undergoing TAVR or SAVR during a 3-year follow-up, but showed a TAVR-related increase in major vascular complications, new pacemaker implantation, and aortic insufficiency and a SAVR-related increased bleeding risk. ${ }^{67}$

The recently published CoreValve trial in high-risk patients was the first to suggest a significantly higher rate of survival at 1 year with TAVR compared with SAVR. In addition to TAVR being non-inferior with respect to echocardiographic indices of valve stenosis, functional status, and quality of life, 1-year survival was superior compared with SAVR (all-cause mortality $14.2 \%$ vs $19.1 \%, P<0.0001$ for non-inferiority, $P=0.04$ for superiority). Within this trial, exploratory analyses also suggested a reduction in the rate of major adverse cardiovascular and cerebrovascular events in TAVR patients. ${ }^{13}$ It is clear that these results must be validated within real-life patient cohorts, which tend to be more diverse than the patients enrolled in rigorous clinical trials.

As main operative outcomes seem to be similar in the bulk of published material, measures such as patient functional status and quality of life are important parameters in considering treatment options, especially in this often elderly and comorbid population. In high-risk patients with severe AS, health status and quality of life have been shown to improve substantially between baseline and 1 year after either TAVR or SAVR. ${ }^{68}$ TAVR via the transfemoral, but not the transapical route, was associated with a shortterm advantage compared with SAVR in this PARTNER subgroup analysis, paving the way for more detailed studies of the "minimalist" 6 or "fast-track"7 TAVR for eligible patients in achieving a prompt and sustainable improvement in functional status and quality of life after relief of AS. 


\section{Patient groups with special considerations}

\section{Patients with previous cardiac surgery}

A redo sternotomy has been identified as an operative risk factor for patients undergoing SAVR ${ }^{69}$ but surprisingly, in a subgroup analysis of the PARTNER cohort, it seemed that SAVR, not TAVR, led to better outcomes in this subgroup..$^{70}$ The TAVR patients demonstrated greater PVL, more frequent repeat hospitalization, increased rate of composite outcome of all-cause mortality and stroke, poorer functional class at 2 years, as well as a trend toward higher mortality at 2 years, after the procedure than SAVR patients. In contrast, a recent institutional study from Emory University showed all-cause 30 -day mortality of $1.9 \%$ for TAVR and $4.1 \%$ for SAVR $(P=0.32)$ in patients with a previous coronary artery bypass graft, a result that marginally favored TAVR after risk adjustment $(P=0.07) .{ }^{71}$ Postoperative morbidity and resource utilization were significantly higher in the SAVR patients, and midterm survival was similar between the two groups after risk adjustment, prompting the question whether the initial findings from the PARTNER trial were more reflective of the lower operative success of the emerging TAVR procedure rather than a true benefit for SAVR patients. Overall, this patient group requires diligent screening by the heart team to identify and weigh the risks of the previous sternotomy.

\section{Patients with renal failure}

Preexisting renal dysfunction is common among patients undergoing SAVR with up to $75 \%$ of patients diagnosed with some level of impairment. ${ }^{72}$ Postoperative AKI is a feared complication that is understandably very prevalent in this subgroup of patients. In SAVR, cardiopulmonary bypass is considered to play a major role in the development of AKI by causing hemodilution, a generalized inflammatory state, as well as hypotension from low pressure and flow rates. In TAVR, some factors contributing to postoperative AKI are the use of contrast agents, hypotension from ventricular rapid pacing, and cholesterol emboli from catheter manipulation. ${ }^{73}$ In a recent meta-analysis of twelve studies including $>90,000$ SAVR patients and 26 studies with $>6,000$ TAVR patients, AKI occurred in 3.4\%-43\% of SAVR cases and in 3.4\%-57\% of TAVR cases, the wide range of incidence due to vastly different definitions of AKI. The 30-day mortality for patients with AKI following SAVR ranged from $5.5 \%$ to $46 \%$, and patients who developed AKI after TAVR had a mortality rate of $7.8 \%-29 \%$, these rates being $2-16$ times higher than in patients without AKI after the procedure. ${ }^{73}$
Some studies comparing SAVR and TAVR in this challenging patient group have demonstrated an advantage of TAVR, showing worsening renal function leading to increased in-hospital mortality, hospital length of stay, and intensive care unit length of stay in SAVR patients, but not in TAVR patients. ${ }^{74}$ Even bearing in mind contrast-induced nephropathy, accepted as a common cause of acute renal failure, ${ }^{75}$ this would suggest that the threshold for utility vs futility is lower for TAVR, and patients with severe renal dysfunction could be considered for TAVR on a case-bycase basis even when ruled inoperable for SAVR. Whether patients of intermediate risk but with a degree of renal dysfunction should be predisposed to TAVR over SAVR is a question to be answered in larger prospective clinical trials specifically targeting this interesting and challenging cohort.

\section{Patients with low ejection fraction}

LV dysfunction portends an increased risk of perioperative mortality in patients undergoing SAVR, ${ }^{76}$ and data from nonrandomized analyses have suggested that TAVR is associated with superior postoperative LV ejection fraction recovery, but similar periprocedural mortality compared with SAVR. ${ }^{77}$ A subgroup analysis from the PARTNER trial demonstrated, however, that mortality rates and LV functional recovery were comparable between valve replacement techniques. ${ }^{78}$ It is interesting to note that in patients with moderate to severe preoperative mitral regurgitation, increased 2-year mortality was observed after SAVR, but not after TAVR, ${ }^{79}$ perhaps suggesting an advantage of TAVR in patients where mitral regurgitation is the etiology behind poor LV function.

\section{Patients with diabetes}

Diabetes is a known risk factor for cardiac surgery interventions and SAVR is no exception. ${ }^{80,81}$ Interestingly, among patients with diabetes and severe symptomatic AS at high risk for surgery, a stratified analysis of the PARTNER trial suggested there is a survival benefit, no increase in stroke, and less renal failure from treatment with TAVR compared with SAVR. ${ }^{40}$ Severe SIRS was associated with a higher mortality after SAVR or TAVR, occurred more commonly after SAVR, and had a greater effect on mortality in patients with diabetes. ${ }^{82}$

Beyond and in addition to diabetes, the contemporary issues of exponentially increasing obesity and metabolic syndrome are extremely intriguing fields to direct future studies as preliminary data seem to indicate that the relationship between these conditions and operative risk do not 
appear identical between SAVR and TAVR. Yamamoto et al recently published a report stating that outcomes were not detrimental in underweight (body mass index $<20$ ) patients undergoing TAVR,${ }^{83}$ while body mass index below normal has been identified as a risk factor for SAVR ${ }^{84}$ Although these findings remain to be validated in larger cohorts, the clinical implications are significant if future studies can show that patients who are obese and diabetic would fare better with one procedure than another.

\section{Cost considerations}

Due to the inherently more minimally invasive nature of TAVR, the issue of potential improved cost-effectiveness has been extensively discussed. Within the PARTNER Trial cohort, 12-month costs and quality-adjusted life years were comparable in both SAVR and TAVR. However, in the subgroup of patients undergoing TAVR via a transfemoral approach, the most minimally invasive access route, TAVR resulted in cost savings of $\sim \$ 1,250$ per patient and led to a modest gain in quality-adjusted life years. ${ }^{85} \mathrm{~A}$ recent European review concluded that in countries with relatively low health-care costs, TAVR is not likely to be cost-effective compared with SAVR in patients with intermediate risk for surgery, mainly because of the high cost of the valve compared with the cost of hospitalization. ${ }^{86}$ However, the authors suggested that TAVR could be cost-effective in specific subgroups and in countries with higher hospitalization costs. In terms of cost-effectiveness, the subgroup of TAVR patients who are eligible for a "minimalist"6 or a "fast-track" transfemoral TAVR are a promising cohort for investigation and may well demonstrate enhanced benefits in this field.

\section{Mid-term and long-term follow-up}

In the longer term, the most comparable data between SAVR and TAVR come from the cohorts of RCTs. The 5-year follow-up of the PARTNER Trial with the first-generation balloon-expandable valve was recently published, reporting 5-year mortality of $67.8 \%$ after TAVR and $62.4 \%$ after SAVR $(P=0.76) .{ }^{87}$ Although strokes at 1 year tended to be more frequent with TAVR $(6.0 \%$ vs $3.2 \%, P=0.08)$, strokes at 5 years were similar for both therapies (TAVR $10.4 \%$ vs SAVR $11.3 \%, P=0.61)$. Echo-derived valve hemodynamics (gradients and areas) were similar for TAVR and SAVR, but moderate or greater aortic regurgitation occurred more frequently in TAVR patients and was associated with increased 5-year risk of mortality. No structural valve deterioration required surgical valve replacement in either group.
Beyond the RCTs, reports from Europe, where TAVR has been used for significantly longer than in the USA, are interesting but suffer from the quick transitions characterizing this novel and rapidly evolving technology. A group from Munich described their TAVR experience in 420 patients over a 3 -year period, reporting that the mean age of patients decreased from 81 to 79 years and the Society of Thoracic Surgeons Predicted Risk of Mortality (STS-PROM) decreased from $7.1 \%$ to $4.8 \%{ }^{88}$ As expected, the 30 -day observed mortality decreased from $11.4 \%$ to $3.8 \%$ at 30 days and 6-month death rate declined from $23.5 \%$ to $12.4 \%$, enforcing the message that the realm of TAVR is quickly changing and outcomes need to be assessed on a rolling basis.

In terms of structural valve deterioration, the results of TAVR patients are still nascent compared with the staggering SAVR experience, which includes studies with up to 30-year follow-up with a reported $99 \%-99.8 \%$ freedom from reoperation for structural valve deterioration in patients aged $>70$ years. ${ }^{89,90}$ While no significant deterioration has been reported thus far, the durability and hemodynamic function of TAVR valves need to be assessed for a considerably longer follow-up period, especially since we expect this technology in the future to be implanted in younger patients.

\section{Establishment of a TAVR program}

As popularity and target audience for TAVR grows, more institutions wish to establish a dedicated TAVR program. The required infrastructure and room requirements have been thoroughly described in a previous article. ${ }^{91}$ Other publications have also described the steps necessary to train not only physicians from various specialties, but the entire supportive and administrative team. ${ }^{92,93}$ This process is currently best summarized by the multisociety consensus documents on TAVR. ${ }^{94,95}$

Like any surgical or interventional procedure, TAVR is found to have a learning curve, which has been quoted to be approximately 30 cases per team. ${ }^{96}$ However, excellent early outcomes have recently been reported by newly initiated relatively low-volume centers, ${ }^{97}$ suggesting that the learning curve may be truncated when a constant integration of lessons learned from larger published experiences is present. ${ }^{98}$ If primary operators receive extensive prior training at highvolume centers, the heart team is appropriately trained and involved, rigorous patient screening is applied, and use of multimodality imaging techniques is routine for the institution, a TAVR program may be established with a diminutive learning curve. ${ }^{98}$ 


\section{Conclusion}

Comparisons between SAVR and TAVR published to date have largely been done between patient cohorts that have at least mid-term follow-up. This means that many patients included in these analyses were operated on in the initial era of TAVR technology and experience. With the constant evolvement of transcatheter valves and delivery systems, as well as the operational and institutional learning curves, it is very likely that the most common current complications of TAVR such as vascular complications and paravalvular leak will diminish in the future.

As TAVR evolves, the target population grows. Trials are underway assessing its use in intermediate-risk populations, while simultaneously modern SAVR is accepting more high-risk patients than a decade ago. This means that the populations are converging and continuous, year-by-year examination of outcomes is warranted. It is likely that TAVR will expand to cover many patients in the intermediate-risk group as the outcomes continue to improve. However, due to the excellent contemporary results of SAVR, there is thus far not enough data to suggest that transcatheter technology would completely replace SAVR.

Despite a stunning number of publications in the field of TAVR and SAVR, no study or registry has yet distilled a flawless algorithm for patient selection between these two operations. To a large extent, we foresee the crucial role of the multispecialty heart team executing a case-by-case assessment of patient selection as the prerequisite of continued operational success regardless of the intervention chosen.

\section{Disclosure}

Dr Thourani is supported with research grants from Edwards Lifesciences, Medtronic, Boston Scientific, and Sorin. He is a consultant for Edwards Lifesciences, Boston Scientific, and St. Jude Medical. The authors report no other conflicts of interest in this work.

\section{References}

1. Go AS, Mozaffarian D, Roger VL, et al; American Heart Association Statistics Committee and Stroke Statistics Subcommittee. Heart disease and stroke statistics - 2013 update: a report from the American Heart Association. Circulation. 2013;127:e6-e245.

2. Leon MB, Smith CR, Mack M, et al; PARTNER Trial Investigators. Transcatheter aortic valve implantation for aortic stenosis in patients who cannot undergo surgery. $N$ Engl J Med. 2010;363: 1597-1607.

3. Shults C, Guyton R, Thourani V. Aortic valve replacement. In: Franco KL, Thourani VH, editors. Cardiothoracic Surgery Review. Philadelphia: Lippincott Williams \& Wilkins; 2012:286-291.
4. Thourani VH, Suri RM, Gunter RL, et al. Contemporary real-world outcomes of surgical aortic valve replacement in 141,905 low-risk, intermediate-risk, and high-risk patients. Ann Thorac Surg. 2015; 99(1):55-61.

5. Bonow RO, Carabello BA, Chatterjee K, et al; 2006 Writing Committee Members, American College of Cardiology/American Heart Association Task Force. 2008 Focused update incorporated into the ACC/AHA 2006 guidelines for the management of patients with valvular heart disease: a report of the American College of Cardiology/American Heart Association Task Force on Practice Guidelines. Circulation. 2008;118(15):e523-e661.

6. Babaliaros V, Devireddy C, Lerakis S, et al. Comparison of transfemoral transcatheter aortic valve replacement performed in the catheterization laboratory (minimalist approach) versus hybrid operating room (standard approach): outcomes and cost analysis. JACC Cardiovasc Interv. 2014;7:898-904.

7. Marcantuono R, Gutsche J, Burke-Julien M, et al. Rationale, development, implementation, and initial results of a fast track protocol for transfemoral transcatheter aortic valve replacement (TAVR). Catheter Cardiovasc Interv. 2015;85(4):648-654.

8. Thourani VH, Iturra S, Sarin EL. Transcatheter aortic valve replacement In: Sellke F, del Nido PJ, Swanson SJ, editors. Sabiston and Spencer Surgery of the Chest. 9th ed. Elsevier; 2015. In press.

9. Brennan JM, Edwards FH, Zhao Y, et al; Developing Evidence to Inform Decisions About Effectiveness-Aortic Valve Replacement (DEcIDE AVR) Research Team. Long-term survival after aortic valve replacement among high-risk elderly patients in the United States: insights from the Society of Thoracic Surgeons Adult Cardiac Surgery Database, 1991 to 2007. Circulation. 2012;126:1621-1629.

10. Durand E, Borz B, Godin M, et al. Performance analysis of EuroSCORE II compared to the original logistic EuroSCORE and STS scores for predicting 30-day mortality after transcatheter aortic valve replacement. Am J Cardiol. 2013;111(6):891-897.

11. Biancari F, Juvonen T, Onorati F, et al. Meta-analysis on the performance of the EuroSCORE II and the Society of Thoracic Surgeons Scores in patients undergoing aortic valve replacement. J Cardiothorac Vasc Anesth. 2014;28(6):1533-1539.

12. Smith CR, Leon MB, Mack MJ, et al; PARTNER Trial Investigators. Transcatheter versus surgical aortic-valve replacement in high-risk patients. N Engl J Med. 2011;364(23):2187-2198.

13. Adams DH, Popma JJ, Reardon MJ. Transcatheter aortic-valve replacement with a self-expanding prosthesis. $N$ Engl J Med. 2014; 370(19):1790-1798.

14. Wenaweser P, Pilgrim T, Kadner A, et al. Clinical outcomes of patients with severe aortic stenosis at increased surgical risk according to treatment modality. J Am Coll Cardiol. 2011;58:2151-2162.

15. Conradi L, Seiffert M, Treede H, et al. Transcatheter aortic valve implantation versus surgical aortic valve replacement: a propensity score analysis in patients at high surgical risk. J Thorac Cardiovasc Surg. 2012;143:64-71.

16. Stöhr R, Dohmen G, Herpertz R, et al. Thirty-day outcome after transcatheter aortic valve implantation compared with surgical valve replacement in patients with high-risk aortic stenosis: a matched comparison. Coron Artery Dis. 2011;22:595-600.

17. Zierer A, Wimmer-Greinecker G, Martens S, Moritz A, Doss M. Is transapical aortic valve implantation really less invasive than minimally invasive aortic valve replacement? J Thorac Cardiovasc Surg. 2009;138:1067-1072.

18. Walther T, Schuler G, Borger MA, et al. Transapical aortic valve implantation in 100 consecutive patients: comparison to propensity-matched conventional aortic valve replacement. Eur Heart J. 2010;31:1398-1403.

19. Holzhey DM, Shi W, Rastan A, Borger MA, Hänsig M, Mohr FW. Transapical versus conventional aortic valve replacement - a propensitymatched comparison. Heart Surg Forum. 2012;15:20111084.

20. De Carlo M, Giannini C, Ettori F, et al. Impact of treatment choice on the outcome of patients proposed for transcatheter aortic valve implantation. EuroIntervention. 2010;6:568-574. 
21. Nielsen $\mathrm{HH}$, Klaaborg KE, Nissen $\mathrm{H}$, et al. A prospective, randomised trial of transapical transcatheter aortic valve implantation vs surgical aortic valve replacement in operable elderly patients with aortic stenosis: the STACCATO trial. EuroIntervention. 2012;8:383-389.

22. Green P, Woglom AE, Genereux P, et al. The impact of frailty status on survival after transcatheter aortic valve replacement in older adults with severe aortic stenosis: a single-center experience. JACC Cardiovasc Interv. 2012;5:974-981.

23. Afilalo J, Alexander KP, Mack MJ, et al. Frailty assessment in the cardiovascular care of older adults. J Am Coll Cardiol. 2014;63(8):747-762.

24. Brown JM, O’Brien SM, Wu C, Sikora JA, Griffith BP, Gammie JS Isolated aortic valve replacement in North America comprising 108,687 patients in 10 years: changes in risks, valve types, and outcomes in the Society of Thoracic Surgeons National Database. J Thorac Cardiovasc Surg. 2009;137(1):82-90.

25. Chikwe J, Adams DH. Frailty: the missing element in predicting operative mortality. Semin Thorac Cardiovasc Surg. 2010;22(2):109-110.

26. Lindman BR, Alexander KP, O'Gara PT, Afilalo J. Futility, benefit, and transcatheter aortic valve replacement. JACC Cardiovasc Interv. 2014;7:707-716.

27. Lee DH, Buth KJ, Martin BJ, Yip AM, Hirsch GM. Frail patients are at increased risk for mortality and prolonged institutional care after cardiac surgery. Circulation. 2010;121(8):973-978.

28. Sündermann S, Dademasch A, Praetorius J, et al. Comprehensive assessment of frailty for elderly high-risk patients undergoing cardiac surgery. Eur J Cardiothorac Surg. 2011;39(1):33-37.

29. Stortecky S, Schoenenberger AW, Moser A, et al. Evaluation of multidimensional geriatric assessment as a predictor of mortality and cardiovascular events after transcatheter aortic valve implantation. JACC Cardiovasc Interv. 2012;5:489-496.

30. Green P, Woglom AE, Genereux P, et al. Gait speed and dependence in activities of daily living in older adults with severe aortic stenosis. Clin Cardiol. 2012;35(5):307-314.

31. Ewe SH, Ajmone Marsan N, Pepi M, et al. Impact of left ventricular systolic function on clinical and echocardiographic outcomes following transcatheter aortic valve implantation for severe aortic stenosis. Am Heart J. 2010;160(6):1113-1120.

32. Bagnall NM, Faiz O, Darzi A, Athanasiou T. What is the utility of preoperative frailty assessment for risk stratification in cardiac surgery? Interact Cardiovasc Thorac Surg. 2013;17(2):398-402.

33. Schoenenberger AW, Stortecky S, Neumann S, et al. Predictors of functional decline in elderly patients undergoing transcatheter aortic valve implantation (TAVI). Eur Heart J. 2013;34:684-692.

34. Noble S, Frangos E, Samaras N, et al. Transcatheter aortic valve implantation in nonagenarians: effective and safe. Eur J Intern Med. 2013;24:750-755

35. Agarwal S, Tuzcu EM, Krishnaswamy A, et al. Transcatheter aortic valve replacement: current perspectives and future implications. Heart. 2015;101(3):169-177.

36. Harken DE, Soroff MS, Taylor MC. Partial and complete prostheses in aortic insufficiency. J Thorac Cardiovasc Surg. 1960;40:744-762.

37. Chiappini B, Camurri N, Loforte A, Di Marco L, Di Bartolomeo R, Marinelli G. Outcome after aortic valve replacement in octogenarians. Ann Thorac Surg. 2004;78(1):85-89.

38. Iturra SA, Suri RM, Greason KL, et al. Outcomes of surgical aortic valve replacement in moderate risk patients: Implications for determination of equipoise in the transcatheter era. J Thorac Cardiovasc Surg. 2014;147:127-132.

39. Généreux P, Cohen DJ, Williams MR, et al. Bleeding complications after surgical aortic valve replacement compared with transcatheter aortic valve replacement: insights from the PARTNER I Trial (Placement of Aortic Transcatheter Valve). J Am Coll Cardiol. 2014;63(11):1100-1109.

40. Lindman BR, Pibarot P, Arnold SV, et al. Transcatheter versus surgical aortic valve replacement in patients with diabetes and severe aortic stenosis at high risk for surgery: an analysis of the PARTNER Trial (Placement of Aortic Transcatheter Valve). J Am Coll Cardiol. 2014;63(11):1090-1099.
41. Mack MJ, Brennan JM, Brindis R, et al; STS/ACC TVT Registry. Outcomes following transcatheter aortic valve replacement in the United States. JAMA. 2013;310(19):2069-2077.

42. Ghatak A, Bavishi C, Cardoso RN, et al. Complications and mortality in patients undergoing transcatheter aortic valve replacement with Edwards SAPIEN and SAPIEN XT Valves: a meta-analysis of world-wide studies and registries comparing the transapical and transfemoral accesses. J Interv Cardiol. 2015;28(3):266-278.

43. Blackstone EH, Suri RM, Rajeswaran J, et al. Propensity-matched comparisons of clinical outcomes after transapical or transfemoral transcatheter aortic valve replacement: a Placement of Aortic Transcatheter Valves (PARTNER)-I Trial Substudy. Circulation. 2015; 131(22):1989-2000.

44. Toggweiler S, Gurvitch R, Leipsic J, et al. Percutaneous aortic valve replacement: vascular outcomes with a fully percutaneous procedure. J Am Coll Cardiol. 2012;59(2):113-118.

45. Généreux P, Head SJ, Van Mieghem NM, et al. Clinical outcomes after transcatheter aortic valve replacement using Valve Academic Research Consortium definitions: a weighted meta-analysis of 3,519 patients from 16 studies. J Am Coll Cardiol. 2012;59:2317-2326.

46. Pilgrim T, Stortecky S, Luterbacher F, Windecker S, Wenaweser P. Transcatheter aortic valve implantation and bleeding: incidence, predictors and prognosis. J Thromb Thrombolysis. 2013;35:456-462.

47. Généreux P, Cohen DJ, Mack M, et al. Incidence, predictors, and prognostic impact of late bleeding complications after transcatheter aortic valve replacement. J Am Coll Cardiol. 2014;64(24):2605-2615.

48. Tamburino C, Capodanno D, Ramondo A, et al. Incidence and predictors of early and late mortality after transcatheter aortic valve implantation in 663 patients with severe aortic stenosis. Circulation. 2011;123(3):299-308.

49. Abdel-Wahab M, Zahn R, Horack M, et al; German transcatheter aortic valve interventions registry investigators. Aortic regurgitation after transcatheter aortic valve implantation: incidence and early outcome. Results from the German transcatheter aortic valve interventions registry. Heart. 2011;97(11):899-906.

50. Ludman PF, Moat N, de Belder MA, et al; UK TAVI Steering Committee and the National Institute for Cardiovascular Outcomes Research. Transcatheter Aortic Valve Implantation in the UK: Temporal Trends, Predictors of Outcome and 6 Year Follow Up: A Report from the UK TAVI Registry 2007 to 2012. Circulation. 2015;131(13):1181-1190.

51. Babaliaros VC, Junagadhwalla Z, Lerakis S, et al. Use of balloon aortic valvuloplasty to size the aortic annulus before implantation of a balloon-expandable transcatheter heart valve. JACC Cardiovasc Interv. 2010;3(1):114-118.

52. Patsalis PC, Al-Rashid F, Neumann T, et al. Preparatory balloon aortic valvuloplasty during transcatheter aortic valve implantation for improved valve sizing. JACC Cardiovasc Interv. 2013;6(9):965-971.

53. Webb J, Gerosa G, Lefèvre T, et al. Multicenter evaluation of a nextgeneration balloon-expandable transcatheter aortic valve. $J$ Am Coll Cardiol. 2014;64(21):2235-2243.

54. Amat-Santos IJ, Dahou A, Webb J, et al. Comparison of hemodynamic performance of the balloon-expandable SAPIEN 3 versus SAPIEN XT transcatheter valve. Am J Cardiol. 2014;114(7):1075-1082.

55. Abdel-Wahab M, Mehilli J, Frerker C, et al; CHOICE investigators. Comparison of balloon-expandable vs self-expandable valves in patients undergoing transcatheter aortic valve replacement: the CHOICE randomized clinical trial. JAMA. 2014;311(15):1503-1514.

56. Rodés-Cabau J. Transcatheter aortic valve implantation: current and future approaches. Nat Rev Cardiol. 2011;9(1):15-29.

57. Jilaihawi H, Chakravarty T, Weiss RE, Fontana GP, Forrester J, Makkar RR. Meta-analysis of complications in aortic valve replacement: comparison of Medtronic-Corevalve, Edwards-Sapien and surgical aortic valve replacement in 8,536 patients. Catheter Cardiovasc Interv. 2012;80(1): $128-138$.

58. Urena M, Webb JG, Tamburino C, et al. Permanent pacemaker implantation after transcatheter aortic valve implantation: impact on late clinical outcomes and left ventricular function. Circulation. 2014;129: 1233-1243. 
59. Hogue CW, Gottesman RF, Stearns J. Mechanisms of cerebral injury from cardiac surgery. Crit Care Clin. 2008;24(1):83-98.

60. Mastoris I, Schoos MM, Dangas GD, Mehran R. Stroke after transcatheter aortic valve replacement: incidence, risk factors, prognosis, and preventive strategies. Clin Cardiol. 2014;37(12):756-764.

61. Marechaux S, Corseaux D, Vincentelli A, et al. Identification of tissue factor in experimental aortic valve sclerosis. Cardiovasc Pathol. 2009; 18:67-76.

62. Alassar A, Soppa G, Edsell M, et al. Incidence and Mechanisms of Cerebral Ischemia After Transcatheter Aortic Valve Implantation Compared With Surgical Aortic Valve Replacement. Ann Thorac Surg. 2015;99(3):802-808.

63. Nietlispach F, Wijesinghe N, Gurvitch R, et al. An embolic deflection device for aortic valve interventions. JACC Cardiovasc Interv. 2010;3:1133-1138.

64. Fanning JP, Walters DL, Platts DG, Eeles E, Bellapart J, Fraser JF. Characterization of neurological injury in transcatheter aortic valve implantation: how clear is the picture? Circulation. 2014; 129(4):504-515.

65. Aguirre J, Waskowski R, Poddar K, et al. Transcatheter aortic valve replacement: experience with the transapical approach, alternate access sites, and concomitant cardiac repairs. JThorac Cardiovasc Surg. 2014; 148(4):1417-1422.

66. Nagaraja V, Raval J, Eslick GD, Ong AT. Transcatheter versus surgical aortic valve replacement: a systematic review and meta-analysis of randomised and non-randomised trials. Open Heart. 2014;1(1): $\mathrm{e} 000013$.

67. Schymik G, Heimeshoff M, Bramlage P, et al. A comparison of transcatheter aortic valve implantation and surgical aortic valve replacement in 1,141 patients with severe symptomatic aortic stenosis and less than high risk. Catheter Cardiovasc Interv. 2015.

68. Reynolds MR, Magnuson EA, Wang K, et al; PARTNER Trial Investigators. Health-related quality of life after transcatheter or surgical aortic valve replacement in high-risk patients with severe aortic stenosis: results from the PARTNER (Placement of AoRTic TraNscathetER Valve) Trial (Cohort A). J Am Coll Cardiol. 2012; 60(6):548-558.

69. Iturra SA, Greason KL, Suri RM, et al. Repeat sternotomy for surgical aortic valve replacement in octogenarian patients with aortic valve stenosis and previous coronary artery bypass graft operation: what is the operative risk? J Thorac Cardiovasc Surg. 2014;148(5):1899-1902.

70. Greason KL, Mathew V, Suri RM, et al. Transcatheter versus surgical aortic valve replacement in patients with prior coronary artery bypass graft operation: a PARTNER trial subgroup analysis. Ann Thorac Surg 2014;98(1):1-8

71. Nguyen TC, Babaliaros VC, Razavi SA, et al. Transcatheter aortic valve replacement has improved short-term but similar midterm outcomes in isolated aortic valve replacement after prior coronary artery bypass grafting. Ann Thorac Surg. 2014;98(4):1316-1324.

72. Thourani VH, Keeling WB, Sarin EL, et al. Impact of preoperative renal dysfunction on long-term survival for patients undergoing aortic valve replacement. Ann Thorac Surg. 2011;91:1798-1807.

73. Najjar M, Salna M, George I. Acute kidney injury after aortic valve replacement: incidence, risk factors and outcomes. Expert Rev Cardiovasc Ther. 2015;16:1-16.

74. Nguyen TC, Babaliaros VC, Razavi SA, et al. Impact of varying degrees of renal dysfunction on transcatheter and surgical aortic valve replacement. J Thorac Cardiovasc Surg. 2013;146(6):1399-1407.

75. Lameire N. The pathophysiology of acute renal failure. Crit Care Clin. 2005;21(2):197-210

76. Halkos ME, Chen EP, Sarin EL, et al. Aortic valve replacement for aortic stenosis in patients with left ventricular dysfunction. Ann Thorac Surg. 2009;88:746-751.

77. Clavel MA, Webb JG, Rodés-Cabau J, et al. Comparison between transcatheter and surgical prosthetic valve implantation in patients with severe aortic stenosis and reduced left ventricular ejection fraction. Circulation. 2010;122:1928-1936.
78. Elmariah S, Palacios IF, McAndrew T, et al; PARTNER Investigators. Outcomes of transcatheter and surgical aortic valve replacement in high-risk patients with aortic stenosis and left ventricular dysfunction: results from the Placement of Aortic Transcatheter Valves (PARTNER) trial (cohort A). Circ Cardiovasc Interv. 2013;6(6):604-614.

79. Barbanti M, Webb JG, Hahn RT, et al; Placement of Aortic Transcatheter Valve Trial Investigators. Impact of preoperative moderate/ severe mitral regurgitation on 2-year outcome after transcatheter and surgical aortic valve replacement: insight from the Placement of Aortic Transcatheter Valve (PARTNER) Trial Cohort A. Circulation. 2013; 128(25):2776-2784

80. Halkos ME, Kilgo P, Lattouf OM, et al. The effect of diabetes mellitus on in-hospital and long-term outcomes after heart valve operations. Ann Thorac Surg. 2010;90:124-130.

81. Smith RL 2nd, Herbert MA, Dewey TM, et al. Does body mass index affect outcomes for aortic valve replacement surgery for aortic stenosis? Ann Thorac Surg. 2012;93:742-746.

82. Lindman BR, Goldstein JS, Nassif ME, et al. Systemic inflammatory response syndrome after transcatheter or surgical aortic valve replacement Heart. 2015;101(7):537-545.

83. Yamamoto M, Hayashida K, Watanabe $\mathrm{Y}$, et al. Effect of body mass index $<20 \mathrm{~kg} / \mathrm{m}$ (2) on events in patients who underwent transcatheter aortic valve replacement. Am J Cardiol. 2015;115(2):227-233.

84. Thourani VH, Keeling WB, Kilgo PD, et al. The impact of body mass index on morbidity and short- and long-term mortality in cardiac valvular surgery. J Thorac Cardiovasc Surg. 2011;142:1052-1061.

85. Reynolds MR, Magnuson EA, Lei Y, et al; PARTNER Investigators. Cost-effectiveness of transcatheter aortic valve replacement compared with surgical aortic valve replacement in high-risk patients with severe aortic stenosis: results of the PARTNER (Placement of Aortic Transcatheter Valves) trial (Cohort A). J Am Coll Cardiol. 2012; 60(25):2683-2692.

86. Ribera A, Slof J, Andrea R, et al. Transfemoral transcatheter aortic valve replacement compared with surgical replacement in patients with severe aortic stenosis and comparable risk: Cost-utility and its determinants. Int J Cardiol. 2014;182C:321-328.

87. Mack MJ, Leon MB, Smith CR, et al; PARTNER 1 Trial Investigators. 5 -year outcomes of transcatheter aortic valve replacement or surgical aortic valve replacement for high surgical risk patients with aortic stenosis (PARTNER 1): a randomised controlled trial. Lancet. 2015 385(9986):2477-2484

88. Lange R, Bleiziffer S, Mazzitelli D, et al. Improvements in transcatheter aortic valve implantation outcomes in lower surgical risk patients: a glimpse into the future. J Am Coll Cardiol. 2012;59(3): 280-287.

89. Forcillo J, Pellerin M, Perrault LP, et al. Carpentier-Edwards pericardial valve in the aortic position: 25-years experience. Ann Thorac Surg. 2013;96:486-493.

90. David TE, Susan Armstrong S, Maganti M. Hancock II bioprosthesis for aortic valve replacement: the gold standard of bioprosthetic valves durability? Ann Thorac Surg. 2010;90:775-781.

91. Kleiman N. Room considerations with TAVR. Methodist Debakey Cardiovasc J. 2012;8(2):19-21.

92. Lauck S, Achtem L, Boone RH, et al. Implementation of processes of care to support transcatheter aortic valve replacement programs. Eur $J$ Cardiovasc Nurs. 2013;12(1):33-38.

93. Bakaeen FG, Kar B, Chu D, et al. Establishment of a transcatheter aortic valve program and heart valve team at a Veterans Affairs facility. Am J Surg. 2012;204(5):643-648.

94. Holmes DR Jr, Mack MJ, Kaul S, et al; American Heart Association, American Society of Echocardiography, European Association for Cardio-Thoracic Surgery, Heart Failure Society of America, Mended Hearts, Society of Cardiovascular Anesthesiologists, Society of Cardiovascular Computed Tomography, Society for Cardiovascular Magnetic Resonance. 2012 ACCF/AATS/SCAI/STS expert consensus document on transcatheter aortic valve replacement. J Thorac Cardiovasc Surg. 2012;144(3):e29-e84. 
95. Tommaso CL, Bolman RM 3rd, Feldman T, et al; American Association for Thoracic Surgery, Society for Cardiovascular Angiography and Interventions, American College of Cardiology Foundation, Society of Thoracic Surgeons. Multisociety (AATS, ACCF, SCAI, and STS) expert consensus statement: operator and institutional requirements for transcatheter valve repair and replacement, part 1: transcatheter aortic valve replacement. J Thorac Cardiovasc Surg. 2012;143(6):1254-1263.

96. Alli OO, Booker JD, Lennon RJ, Greason KL, Rihal CS, Holmes DR Jr. Transcatheter aortic valve implantation: assessing the learning curve. JACC Cardiovasc Interv. 2012;5(1):72-79.
97. Chevalier F, Poulin F, Lamarche Y, et al. Excellent outcomes for transcatheter aortic valve replacement within 1 year of opening a lowvolume centre and consideration of requirements. Can J Cardiol. 2014; 30(12):1576-1582.

98. Nazif TM, Leon MB. Starting small but aiming high: important lessons in establishing a successful TAVR program. Can J Cardiol. 2014; 30(12):1506-1507.

\section{Publish your work in this journal}

Research Reports in Clinical Cardiology is an international, peerreviewed, open access journal publishing original research, reports, editorials, reviews and commentaries on all areas of cardiology in the clinic and laboratory. The manuscript management system is completely online and includes a very quick and fair peer-review system.
Visit http://www.dovepress.com/testimonials.php to read real quotes from published authors. 